\title{
MRI-detectable polymeric micelles incorporating platinum anticancer drugs enhance survival in an advanced hepatocellular carcinoma model
}

This article was published in the following Dove Press journal:

International Journal of Nanomedicine

25 June 2015

Number of times this article has been viewed

\author{
Nguyen Quoc Vinh' \\ Shigeyuki Naka' \\ Horacio Cabral ${ }^{2}$ \\ Hiroyuki Murayama' \\ Sachiko Kaida' \\ Kazunori Kataoka ${ }^{2}$ \\ Shigehiro Morikawa ${ }^{3}$ \\ Tohru Tani ${ }^{4}$ \\ 'Department of Surgery, Shiga \\ University of Medical Science, Shiga, \\ Japan; ${ }^{2}$ Department of Bioengineering, \\ Graduate School of Engineering, \\ The University of Tokyo, Tokyo, \\ Japan; ${ }^{3}$ Department of Nursing, Shiga \\ University of Medical Science, Shiga, \\ Japan; ${ }^{4}$ Biomedical Innovation Center, \\ Shiga University of Medical Science, \\ Shiga, Japan
}

Correspondence: Shigeyuki Naka

Department of Surgery, Shiga University of Medical Science, Seta-Tsukiniwa,

Otsu, Shiga, 520-2192, Japan

$\mathrm{Tel}+81775482238$

Fax +8I 775482240

Email naka@belle.shiga-med.ac.jp
Abstract: Hepatocellular carcinoma (HCC) is one of the most intractable and lethal cancers; most cases are diagnosed at advanced stages with underlying liver dysfunction and are frequently resistant to conventional chemotherapy and radiotherapy. The development of tumor-targeting systems may improve treatment outcomes. Nanomedicine platforms are of particular interest for enhancing chemotherapeutic efficiency, and they include polymeric micelles, which enable targeting of multiple drugs to solid tumors, including imaging and therapeutic agents. This allows concurrent diagnosis, targeting strategy validation, and efficacy assessment. We used polymeric micelles containing the T1-weighted magnetic resonance imaging contrast agent gadoliniumdiethylenetriaminpentaacetic acid (Gd-DTPA) and the parent complex of the anticancer drug oxaliplatin [(1,2-diaminocyclohexane)platinum(II) (DACHPt)] for simultaneous imaging and therapy in an orthotopic rat model of HCC. The Gd-DTPA/DACHPt-loaded micelles were injected into the hepatic artery, and magnetic resonance imaging performance and antitumor activity against HCC, as well as adverse drug reactions were assessed. After a single administration, the micelles achieved strong and specific tumor contrast enhancement, induced high levels of tumor apoptosis, and significantly suppressed tumor size and growth. Moreover, the micelles did not induce severe adverse reactions and significantly improved survival outcomes in comparison to oxaliplatin or saline controls. Our results suggest that Gd-DTPA/DACHPt-loaded micelles are a promising approach for effective diagnosis and treatment of advanced HCC.

Keywords: Gd-DTPA/DACHPt-loaded micelles, polymeric micelles, drug delivery, hepatocellular carcinoma

\section{Introduction}

The incidence of hepatocellular carcinoma (HCC), a leading cause of cancer death worldwide, is gradually increasing. ${ }^{1,2}$ Because most patients with $\mathrm{HCC}$ are diagnosed with intermediate or advanced-stage disease, ${ }^{3}$ only a small proportion of these patients are eligible for treatment with curative intent, which is usually accomplished through percutaneous ablation or partial hepatectomy and liver transplantation. Moreover, although several therapeutic strategies have been proposed, ${ }^{4-6}$ the survival outcomes of patients with advanced HCC have not improved apace.

Nanoparticle drug carriers have been demonstrated as an effective method of targeting drugs to malignant tumors with permeable neovasculature and impaired lymphatic drainage, the so-called enhanced permeability and retention (EPR) effect. $^{7,8}$ Therefore, imaging and chemotherapeutic agents incorporated within these carriers can selectively accumulate in tumors at high concentrations, thus increasing the detection of specific tumors and improving antitumor activity while reducing 
adverse reactions. ${ }^{9-11}$ In recent years, several nanoscale drug-carrier modalities have been developed, including liposomes, dendrimers, polymer-drug conjugates, and polymeric micelles. ${ }^{10,12-16}$ Polymeric micelles are generated by the selfassembly of amphiphilic block copolymers into core-shell nanostructures with drug-loaded cores and biocompatible shells and have demonstrated advantages as nanoparticle drug carriers. They are relatively small (10-100 nm), can incorporate various substances with controlled release, exhibit prolonged circulation in the bloodstream, and selectively accumulate in cancerous tissues. ${ }^{17-21}$ Several micelle formations with anticancer agents, doxorubicin (NK911), paclitaxel (NK105), SN-38 (NK012), cisplatin (NC6004), and (1,2diaminocyclohexane)platinum(II) (DACHPt) (NC4016), are undergoing clinical trial and have demonstrated significant anticancer efficacy and fewer adverse reactions than that with the use of free drugs. ${ }^{22-27}$ Moreover, the integration of tracer functions for clinical imaging, including computed tomography, magnetic resonance imaging (MRI), single-photon emission computed tomography, and positron emission tomography, with anticancer drugs allows the construction of polymeric micelle carriers for simultaneous diagnosis and therapy. $9,20,28,29$ In clinical practice, the ability to combine such imaging substances and antitumor drugs is extremely advantageous because it would permit observation of drug delivery to the tumor, thus enabling clinicians to identify effective dose regimens and anticipate treatment efficacy.

We recently developed MRI-detectable polymeric micelles for simultaneous diagnosis and therapy by incorporating the T1-weighted MRI contrast agent gadoliniumdiethylenetriaminpentaacetic acid (Gd-DTPA) and the platinum anticancer drug DACHPt, the parent complex of the anticancer drug oxaliplatin, within the cores of micelles (Figure 1). Earlier characterization of these micelles revealed a relatively small diameter of $33 \mathrm{~nm}$, which allowed high penetration and accumulation even in tumors with poor permeability. ${ }^{17,30}$ In this study, we examined the feasibility of using these Gd-DTPA/DACHPt-loaded micelles to simultaneously diagnose and treat a clinically relevant rat model of HCC that was generated using the N1-S1 hepatoma cell line, which exhibits high levels of malignancy and hypervascularization. After injecting the micelles through

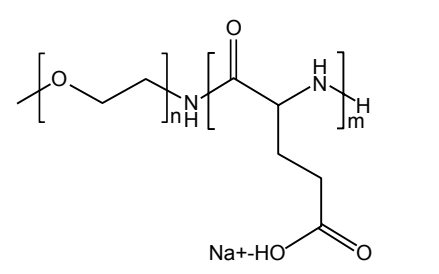

Poly(ethylene glycol)-b-poly(glutamic acid) [PEG-b-P(Glu)]

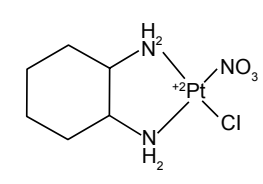

(1,2-diaminocyclohexane)platinum(II) (DACHPt) nitrate chloride

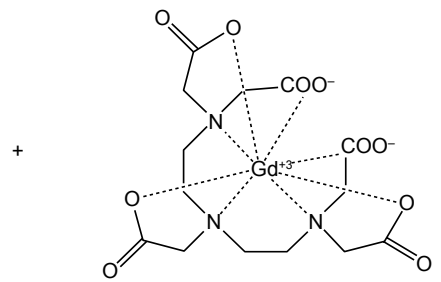

Gadolinium diethylenetriaminpentaacetic acid (Gd-DTPA)

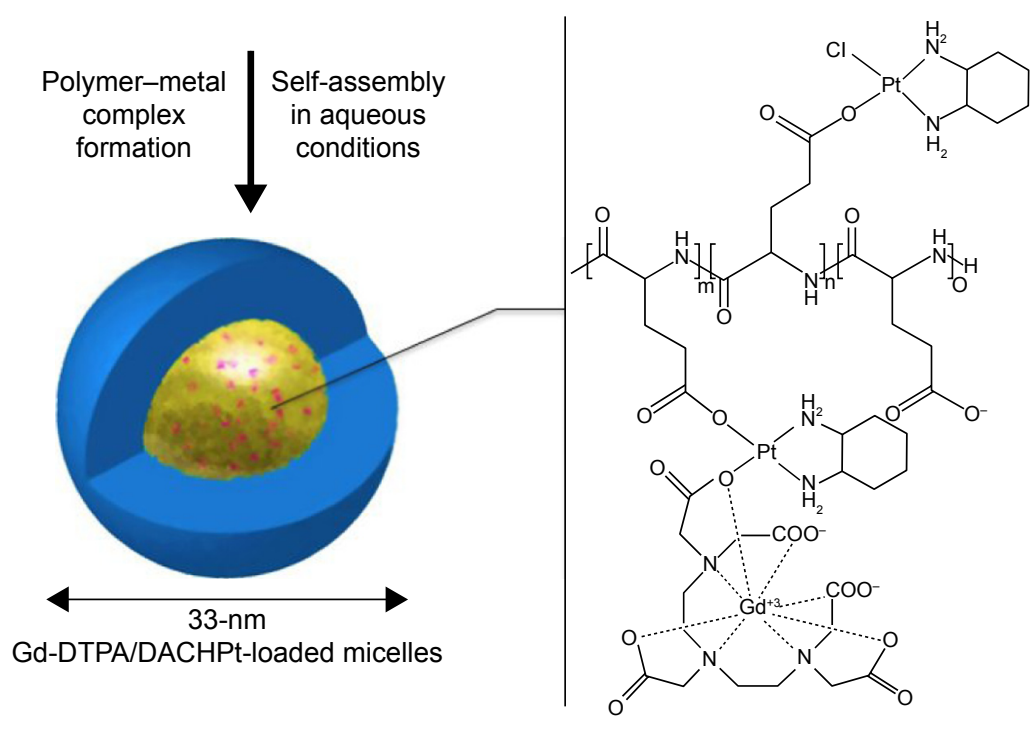

Figure I Schematic diagram of self-assembly of Gd-DTPA/DACHPt-loaded micelles.

Notes: MRI-detectable polymeric micelles for simultaneous diagnosis and therapy are created by incorporating the TI-weighted MRI contrast agent Gd-DTPA and the platinum anticancer drug DACHPt nitrate chloride, which is the parent complex of the anticancer drug oxaliplatin, within the micelle cores. 
the hepatic artery, we performed MRI, tumor cytotoxicity, biochemistry, and tumor progression analyses to investigate MRI performance, adverse reactions, and antitumor activity of the micelles. Our results demonstrated the safety and enhanced efficacy of Gd-DTPA/DACHPt-loaded micelles for the selective imaging and treatment of HCC.

\section{Materials and methods}

The experimental protocol was approved by the Committee on Animal Care of Shiga University of Medical Science, and all experiments were performed in accordance with institutional guidelines.

\section{Preparation and verification of Gd-DTPA/ DACHPt-loaded micelles}

The poly(ethylene glycol)- $b$-poly(glutamic acid) [PEG$b$-P(Glu) $]\left[\mathrm{Mw}_{\mathrm{PEG}}=12,000 \mathrm{Da}\right.$; polymerization degree of $\mathrm{P}(\mathrm{Glu})=20$ ] block copolymer was synthesized according to a previously described synthetic method. ${ }^{31}$ Briefly, the $N$-carboxyanhydride of $\gamma$-benzyl L-glutamate was synthesized by the Fuchs-Farthing method using triphosgene. Then, the $N$-carboxyanhydride of $\gamma$-benzyl L-glutamate was polymerized in DMF, initiated by the primary amino group of $\mathrm{CH}_{3} \mathrm{O}-\mathrm{PEG}-\mathrm{NH}_{2}$, to obtain PEG- $b$-poly $(\gamma$-benzylL-glutamate) (PEG- $b$-PBLG). The polymerization degree was verified by comparing the proton ratios of methylene units in PEG $\left(-\mathrm{OCH}_{2} \mathrm{CH}_{2}-: \delta=3.7 \mathrm{ppm}\right)$ and phenyl groups of PBLG $\left(-\mathrm{CH}_{2} \mathrm{C}_{6} \mathrm{H}_{5}, \delta=7.3 \mathrm{ppm}\right)$ by ${ }^{1} \mathrm{H}$ nuclear magnetic resonance (NMR) measurement (solvent, DMSO-d $\mathrm{d}_{6}$; JEOL EX270, JEOL, Inc., Tokyo, Japan). PEG- $b$-PBLG was deprotected by mixing with $0.5 \mathrm{~N} \mathrm{NaOH}$ at room temperature to obtain PEG- $b$-P(Glu). Complete deprotection was confirmed by ${ }^{1} \mathrm{H}-\mathrm{NMR}$ measurement (solvent, $\mathrm{D}_{2} \mathrm{O}$; temperature, $25^{\circ} \mathrm{C}$ ).

The Gd-DTPA/DACHPt-loaded micelles were created by self-assembling as described. ${ }^{17}$ Briefly, Gd-DTPA (SigmaAldrich Japan, Tokyo, Japan) was converted to sodium salt by adjusting the $\mathrm{pH}$ to 7 with $\mathrm{NaOH}$, and lyophilized. A $5 \mathrm{mmol} / \mathrm{L}$ solution of bis(nitrato) (trans-L-1,2-diaminocyclohexane)platinum(II) [DACHPt $\left(\mathrm{NO}_{3}\right)_{2}$; W.C. Heraeus $\mathrm{GmbH} \& \mathrm{Co} . \mathrm{KG}$, Hanau, Germany] in water was mixed with the sodium salt of Gd-DTPA ( $5 \mathrm{mmol} / \mathrm{L})$, and the solution was maintained for 24 hours at $37^{\circ} \mathrm{C}$. Then, PEG- $b-\mathrm{P}(\mathrm{Glu})$ ([Glu $]=5 \mathrm{mmol} / \mathrm{L}$ ) was added to this solution ([DACHPt $] /$ [Glu $]=1.0$ ) and are allowed to react for 120 hours at $37^{\circ} \mathrm{C}$ to prepare Gd-DTPA/DACHPt-loaded micelles. The micelles were dialyzed for 24 hours with Milli-Q water, which was exchanged five times [molecular weight cutoff size (MWCO): 2,000; Spectra/Por-6, Spectrum Laboratories,
Rancho Dominguez, CA, USA] and followed by ultrafiltration (MWCO: 30,000).

The size distribution of the Gd-DTPA/DACHPt-loaded micelles was evaluated by dynamic light scattering measurement at $25^{\circ} \mathrm{C}$ using a Zetasizer Nano ZS90 (Malvern Instruments, Malvern, UK). The encapsulation of DACHPt and Gd-DTPA inside the micelles was determined by inductively coupled plasma mass spectrometry (ICP-MS; 4500 ICP-MS, Hewlett Packard). The absence of $\mathrm{Gd}^{3+}$ in the Gd-DTPA/ DACHPt mixture was confirmed by using the arsenazo III method. ${ }^{32}$ The stability of Gd-DTPA/DACHPt-loaded micelles under physiological conditions was determined by DLS and static light scattering using a Zetasizer Nano ZS90 (Malvern Instruments).

The MR contrast effect of the magnetic nanoparticles was examined by measuring their proton longitudinal relaxivities, $r_{1}$, the definition of which is the slope of the concentration dependence given as $1 / T_{1}=1 / T_{10}+r_{1}[\mathrm{Gd}]$, where $T_{1}$ is the longitudinal relaxation time, $1 / T_{1}$ is the longitudinal relaxation rate contrast in the presence of a paramagnetic species, and $1 / T_{10}$ is the longitudinal relaxation rate contrast in the absence of a paramagnetic species.

Morphology of Gd-DTPA/DACHPt-loaded micelles was observed by transmission electron microscopy (Hitachi H-7000, Hitachi Ltd., Tokyo, Japan) at an accelerating voltage of $75 \mathrm{kV}$. The micelles $(1 \mu \mathrm{L})$ were placed on 400 -mesh copper grids coated with a thin film of collodion followed by carbon. The samples were stained by the deposition of a drop of an aqueous solution of $50 \%$ ethanol and $2 \mathrm{wt} \%$ uranyl acetate.

\section{Cancer cell lines and the HCC model}

The N1-S1 rat hepatoma cell line was kindly provided by HPA Culture Collections (USA). The cells were cultured in RPMI 1640 medium (Sigma-Aldrich) containing 10\% fetal bovine serum at $37^{\circ} \mathrm{C}$ in a humidified atmosphere containing 5\% $\mathrm{CO}_{2}$. F344/N rnu/rnu nude rats (9-week-old males; body weight, 240-270 g) and Sprague Dawley rats (10-week-old males; body weight, 300-350 g) were purchased from CLEA, Tokyo, Japan. Each rat was anesthetized at an induction dose of 5\% isoflurane in a medical oxygen mixture and maintained at $1.5 \%-2.5 \%$ isoflurane. A minilaparotomy of the upper abdomen was performed to expose the liver. The external left lobe was subserosally injected with $1 \times 10^{6} \mathrm{~N} 1-\mathrm{S} 1$ hepatoma cells $\left(25 \mu \mathrm{L}\right.$ of $4 \times 10^{7}$ cells $/ \mathrm{mL}$ suspension). The abdominal incision was subsequently closed, and the rat was returned to the animal facility. After 6 or 7 days, the rats were again anesthetized. After confirmation 
of tumor formation, a polyethylene catheter (inner and outer diameters of 0.28 and $0.61 \mathrm{~mm}$, respectively) was inserted retrogressively into the gastroduodenal artery, with the tip placed at the proper hepatic artery, as described. ${ }^{33} \mathrm{Gd}-$ DTPA/DACHPT-loaded micelles, oxaliplatin, free GdDTPA (Magnevist ${ }^{\circledR}$, Bayer AG, Leverkusen, Germany), or saline was injected into the hepatic artery via the catheter. MRI performance, histology, and antitumor activity were subsequently examined.

\section{MRI performance of Gd DTPA/DACHPt- loaded micelles}

This experiment was performed in N1-S1 tumor-bearing Sprague Dawley rats. After general anesthesia and hepatic artery catheterization, each rat was fixed on an acrylic cradle and a respiratory sensor was attached to the chest wall to allow monitoring of respiration during MRI. MR images were obtained using a GE Signa 1.5 T MRI (GE Healthcare UK Ltd, Little Chalfont, UK) equipped with a 5-inch GP RF surface coil (model: 46-258773G1). The following T1-weighted gradient echo (T1-GE) imaging parameters were used: repetition time $=14.7 \mathrm{~ms}$, echo time $=3.3 \mathrm{~ms}$, flip angle $=30^{\circ}$, field of view $=80 \times 80 \mathrm{~mm}^{2}$, matrix size $=256 \times 224$, and axial slice thickness $=2 \mathrm{~mm}$. The following T1-weighted spin echo (T1-SE) parameters were used: repetition time $=600 \mathrm{~ms}$, echo time $=15.4 \mathrm{~ms}$, flip angle $=30^{\circ}$, field of view $=80 \times 80 \mathrm{~mm}^{2}$, matrix size $=256 \times 256$, and axial slice thickness $=2 \mathrm{~mm}$. Each rat was injected via the hepatic artery with a $2.5 \mathrm{mg} / \mathrm{kg}$ Gd-DTPA-equivalent dose of GdDTPA/DACHPt-loaded micelles. MR images were acquired immediately after injection and every 10 minutes thereafter, while alternating between T1-GE and T1-SE for a total duration of 180 minutes. Identical experiments were conducted after injecting $2.5 \mathrm{mg} / \mathrm{kg}$ free Gd-DTPA (Magnevist ${ }^{\circledR}$ ) or DACHPt-loaded micelles (without Gd-DTPA) with an equivalent Pt dose as controls. All rats were sacrificed after completion of MRI to confirm the tumor location on imaging with macroscopic observation. The tumors were then excised, fixed in 10\% formaldehyde, paraffin-embedded, and stained with hematoxylin and eosin (H\&E) for histology. The MR images were analyzed with ImageJ (version 1.46r, NIH, Bethesda, MD, USA) and Excel software (Microsoft, Inc., Redmond, WA, USA). The region of interest, which included the tumor or healthy liver, was drawn on the same slice level at each time point. The mean pixel grayscale value indicated the region-of-interest intensity, which was compared in the tumor or healthy liver to the precontrast images and the water phantom.
Tumor cytotoxicity and therapeutic effect N1-S1 tumor-bearing F344/N rnu/rnu rats were treated with an $8 \mathrm{mg} / \mathrm{kg}$ DACHPt-equivalent dose of micelles, $8 \mathrm{mg} / \mathrm{kg}$ oxaliplatin, or an identical volume of saline. These rats were separated into two groups for the tumor cytotoxicity study and tumor size evaluation after treatment. To evaluate tumor cytotoxicity, the rats were sacrificed 3 days postinjection and the tumors were harvested. The samples were fixed in $4 \%$ paraformaldehyde and paraffin-embedded, and two adjacent $3 \mu \mathrm{m}$ sections were cut. One section was used for $\mathrm{H} \& \mathrm{E}$ staining and the other was subjected to terminal deoxynucleotidyl transferase dUTP nick-end labeling (TUNEL) to assess apoptosis. The TUNEL assay was performed according to the manufacturer's protocol (Promega DeadEND Fluorometric TUNEL system, Promega Corporation, Fitchburg, WI, USA). Nuclei were counterstained with 4',6-diamidino-2-phenylindole (Vector Laboratories, Burlingame, CA, USA). We used a Nanozoomer (2.0-RS C10730-SG, Hamamatsu Photonics, Shizuoka, Japan) to capture images of the entire tumoral areas of the H\&Estained samples at $20 \times$ magnification. These images, along with the fluorescence observation of TUNEL-stained slices, were observed simultaneously in order to define the apoptotic regions within the tumors. The areas of the apoptotic regions were calculated with NDP viewer (supported by Hamamatsu), and the apoptotic area percentage was calculated as follows: the total apoptotic area (TUNEL-positive staining) divided by the tumor area.

Therapeutic efficacy was assessed by measuring tumor size after treatment. Before treatment and at 3,6, 10, 14, and 21 days postinjection, T1-GE MR images were obtained with the following parameters: repetition time $=8.9 \mathrm{~ms}$, echo time $=2.1 \mathrm{~ms}$, flip angle $=30^{\circ}$, field of view $=80 \times 80 \mathrm{~mm}^{2}$, matrix size $=128 \times 128$, axial slice thickness $=1.0 \mathrm{~mm}$, and space $=0.0 \mathrm{~mm}$. Tumor reconstruction and volume calculations were performed using OxiriX (version 5.0.2, Pixmeo Sarl, Bernex, Geneva, Switzerland). These rats were followed up until death to record survival outcomes.

\section{Blood test examination}

Hepatic and renal functions were tested for partial evaluation of adverse reactions after hepatic arterial injection of Gd DTPA/DACHPt-loaded micelles, oxaliplatin, or saline. Blood was harvested from the tail veins and assayed using VetScan (PC-2001; Abaxis, Inc., Sunnyvale, CA, USA) and a blood test kit (A500-0038-H, Abaxis, Inc.) before injection and at 1 day, 1 week, and 2 weeks postinjection. 


\section{Statistical analysis}

Statistical analysis was performed using the PRISM version 5 statistical software package (GraphPad Software Inc., La Jolla, CA, USA). Statistical differences were evaluated by one-way analysis of variance and Bonferroni's multiple comparison test. $P$-values $<0.05$ were considered significant.

\section{Results \\ Characterization of Gd-DTPA/DACHPt- loaded micelles}

The resulting micelles had a core-shell structure, in which the inner core loaded with Gd-DTPA/DACHPt was surrounded by an outer shell of PEG palisade (Figures 1 and 2). These micelles were $33 \mathrm{~nm}$ in diameter, with a narrow size distribution. The micelles incorporated $0.42 \mathrm{mg}$ of DACHPt per milligram polymer and $0.04 \mathrm{mg}$ of Gd-DTPA per milligram polymer, corresponding to $45 \%$ and $5 \%$ of the carboxylic groups in PEG- $b$-P(Glu), respectively. The $r_{1}$ of the micelles increased to $80.7 \mathrm{mmol} / \mathrm{L} / \mathrm{s}, 24$-fold greater than that of Gd-DTPA alone. No free $\mathrm{Gd}^{3+}$ was detected in these micelles, indicating that the safety of Gd-DTPA chelates remained stable.

\section{Gd-DTPA/DACHPt-loaded micelle- mediated tumor enhancement}

The T1-weighted MR images (T1-GE and T1-SE) before the hepatic arterial injection of Gd-DTPA/DACHPt-loaded

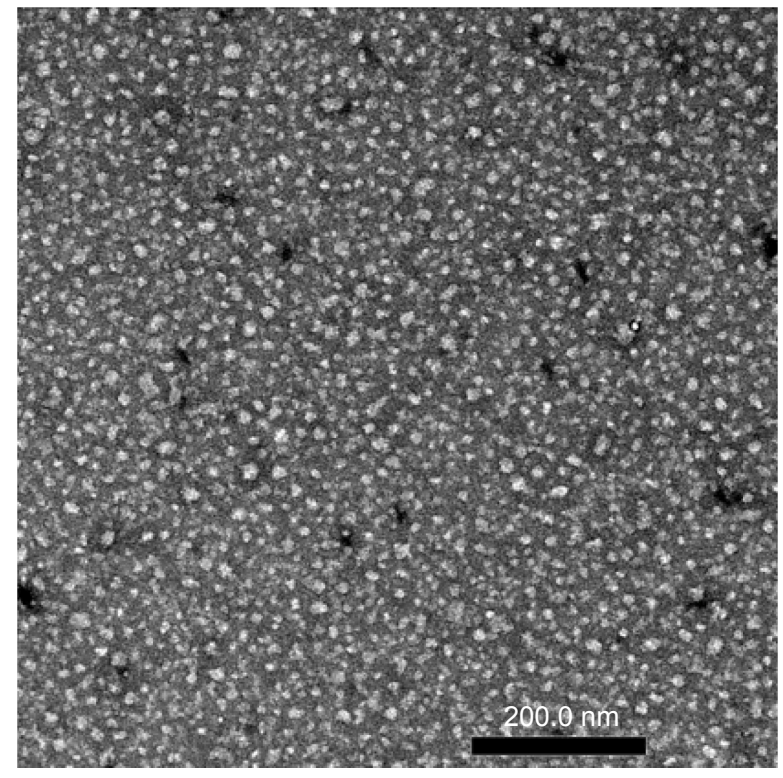

Figure 2 Transmission electron microscopy image of Gd-DTPA/DACHPt-loaded micelles, the Gd-DTPA/DACHPt-loaded micelles come out as white spots. Abbreviations: DACHPt, (I,2-diaminocyclohexane)platinum(II); Gd-DTPA, gadoliniumdiethylenetriaminpentaacetic acid. micelles showed lower signal intensity in the tumor than in the surrounding healthy liver. After injection of Gd-DTPA/ DACHPt-loaded micelles $(\mathrm{n}=9)$, the signal intensity of the tumor increased considerably to a level greater than that of the surrounding liver on both T1-GE and T1-SE images, whereas the signal intensity of the healthy liver remained near the initial values (Figure 3A and B). Moreover, the MR images collected every 10 minutes showed that the tumor contrast enhancement remained stable for up to 3 hours, thus indicating retention of the micelles in the HCC tissues. Because hydrophilic Gd-DTPA is gradually released from the micelles, the signal began to slowly decrease at 3 hours postinjection, likely matching the discharge and clearance of Gd-DTPA from the tumor. It is worth noting that tumor enhancement was heterogeneous, and darker spots were observed within the tumors, perhaps related to necrotic regions without blood flow. No tumor contrast enhancement was observed with the injection of free Gd-DTPA $(n=9)$ or DACHPt micelles $(n=2)$, and the tumor intensities remained lower than that of the healthy liver with both control treatments (Figure 3C-F). Moreover, the macroscopic observations after MRI acquisition confirmed the tumor positions (Figure 4A), and histology revealed malignant cells with hyperchromatic nuclei, a high nuclear/cytoplasmic ratio, poor differentiation, hypervascularization, and scattered necrotic regions, supporting our hypothesis regarding the darker regions in the micelle-enhanced tumor contrast areas on MRI (Figure 4B and C).

\section{In vivo cytotoxicity against tumor cells}

Three days after injecting the micelles, oxaliplatin, or saline, the microscopic observation of H\&E-stained tumor sections revealed two different regions: one comprised typical malignant cells and the other comprised disrupted cells characterized by missing nuclei, indistinct cell walls, or nuclei without surrounding cell structures. Collation with the fluorescent TUNEL assay results showed that these disrupted regions corresponded to regions with high levels of positive fluorescein-12-dUTP incorporation (TUNELpositive; Figure 4D and E). We observed a significant difference in apoptotic area in tumors treated with the micelles, oxaliplatin, or saline (Figure 5). The mean tumor apoptosis percentages at 3 days postinjection were $92.4 \% \pm 3.4 \%$ with the micelles $(\mathrm{n}=10), 45.3 \% \pm 20.8 \%$ with oxaliplatin $(\mathrm{n}=10)$, and $9.65 \% \pm 5.9 \%$ with saline $(\mathrm{n}=10)$. These results clearly showed that Gd DTPA/DACHPt-loaded micelles induced tumor cell death much more efficiently than free oxaliplatin and saline $(P<0.001)$. 
A

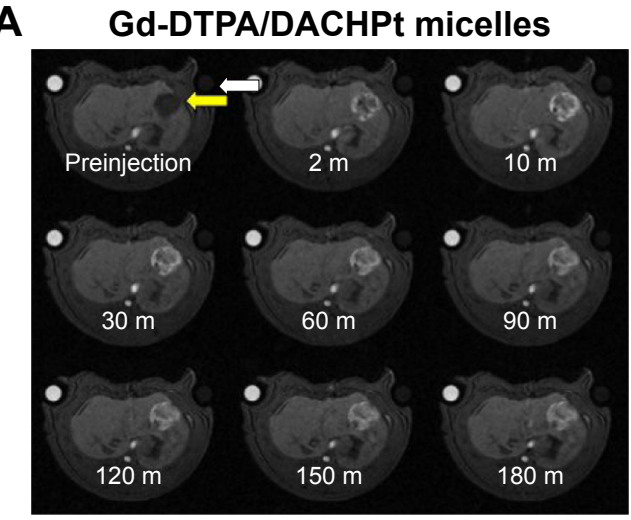

C

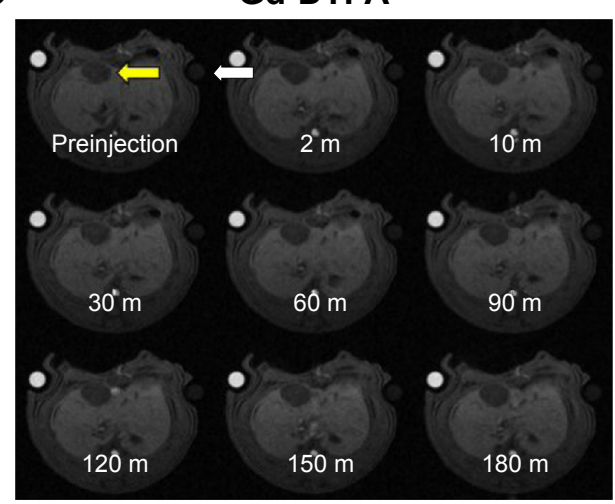

E

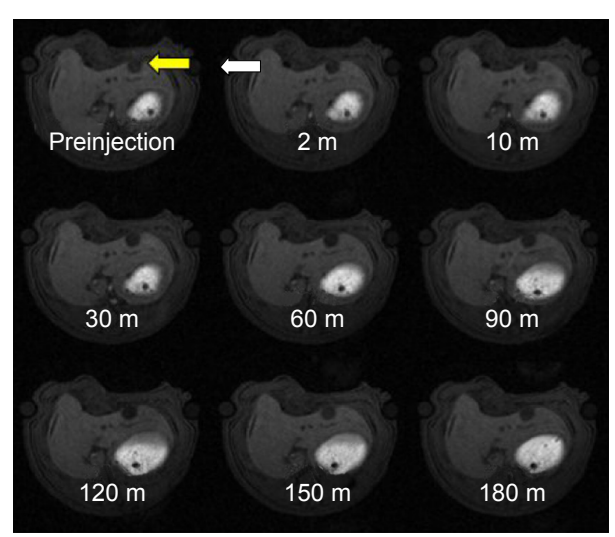

$\mathbf{B}$

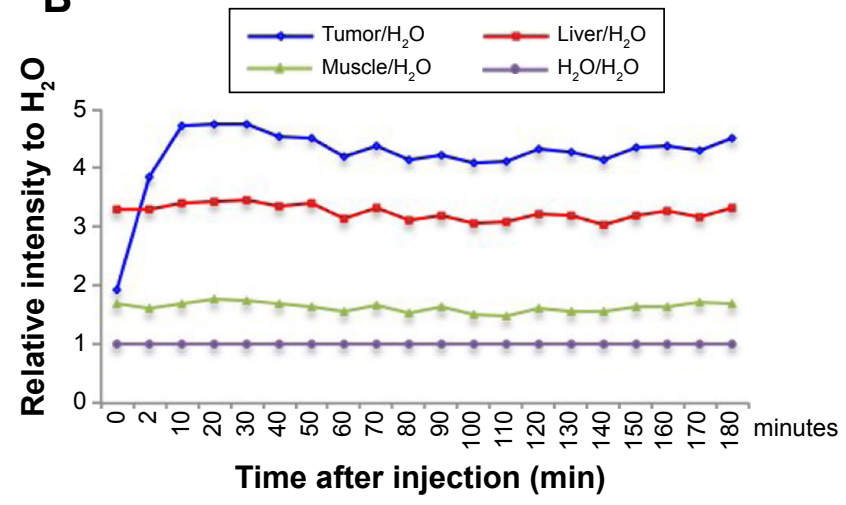

D

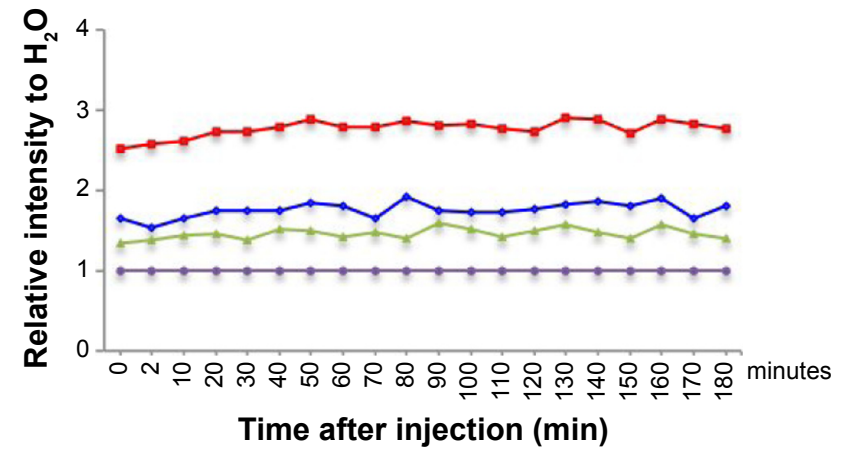

$\mathbf{F}$

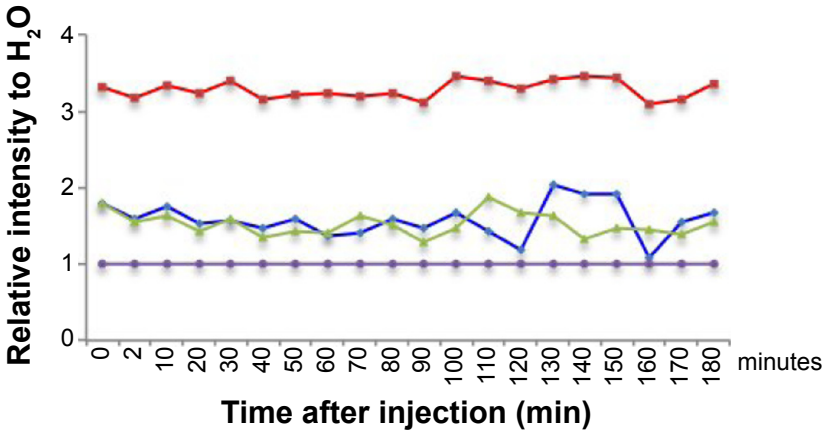

Figure $3 \mathrm{MRI}$ performance of Gd-DTPA/DACHPt-loaded micelles in an NI-SI hepatic tumor (yellow arrow).

Notes: (A) TI-GE MRI series after the injection of Gd-DTPA/DACHPt-loaded micelles. The MR images collected at 2 minutes and every 10 minutes after the injection showed that the tumor contrast enhancement remained stable for up to 3 hours. (B) The signal intensity of the tumor increased considerably to a level greater than that of the surrounding liver, whereas the signal intensity of the healthy liver remained near the initial values. (C, E) TI-GE MRI series after the injection of free Gd-DTPA or DACHPt micelles, any enhancement of the contrast was not observed in the tumor. (D, F) The tumor intensities remained lower than that of the healthy liver with both control treatments. A tube containing $\mathrm{H}_{2} \mathrm{O}$ was set at the upper right as a reference (white arrows).

Abbreviations: DACHPt, (I,2-diaminocyclohexane)platinum(II); Gd-DTPA, gadolinium-diethylenetriaminpentaacetic acid; TI-GE MRI, TI-weighted gradient echo magnetic resonance imaging.

\section{Tumor growth suppression and animal survival}

The MR images were obtained at a slice thickness of $1 \mathrm{~mm}$ and a spacing of $0 \mathrm{~mm}$ to allow accurate volume calculations. Figure 6 shows tumor growth after injecting Gd-DTPA/ DACHPt-loaded micelles, oxaliplatin, or saline. At the time of injection, the mean tumor volumes were $231 \mathrm{~mm}^{3}$ in the micelle group ( $\mathrm{n}=6), 172 \mathrm{~mm}^{3}$ in the oxaliplatin group $(\mathrm{n}=6)$, and $144 \mathrm{~mm}^{3}$ in the saline group $(\mathrm{n}=5)$. At 3 days postinjection, the mean tumor volume of the micelle group decreased to $171 \mathrm{~mm}^{3}$ ( $74.0 \%$ of the preinjection volume), and this tumor suppression continued until 10 days postinjection, at which time the mean tumor volume was $99.1 \%$ of the preinjection volume. The tumors continued to grow and 
A

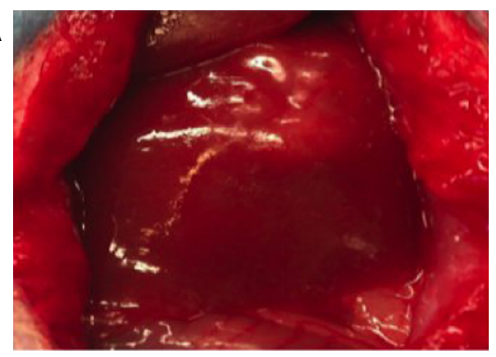

B

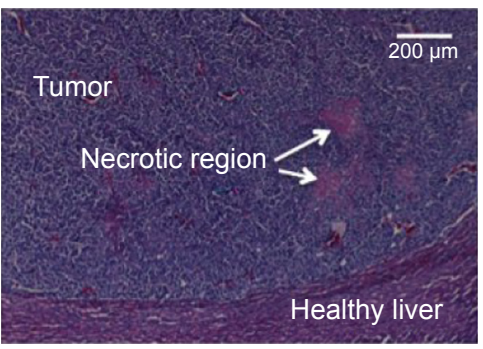

C

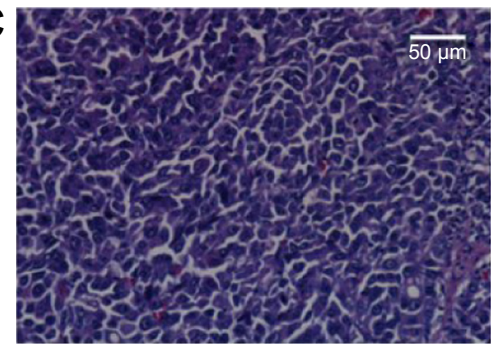

D

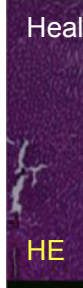

Healthy liver
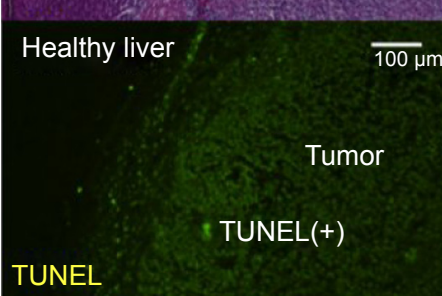

Healthy liver

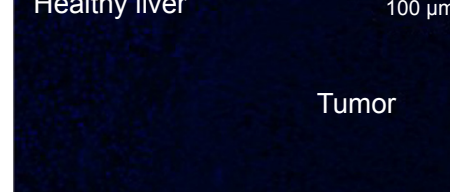

DAPI

Healthy liver

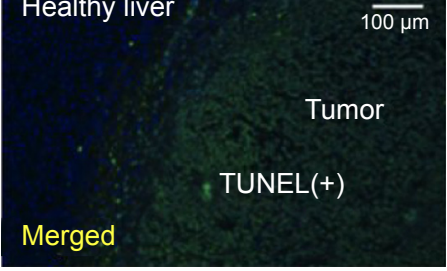

E

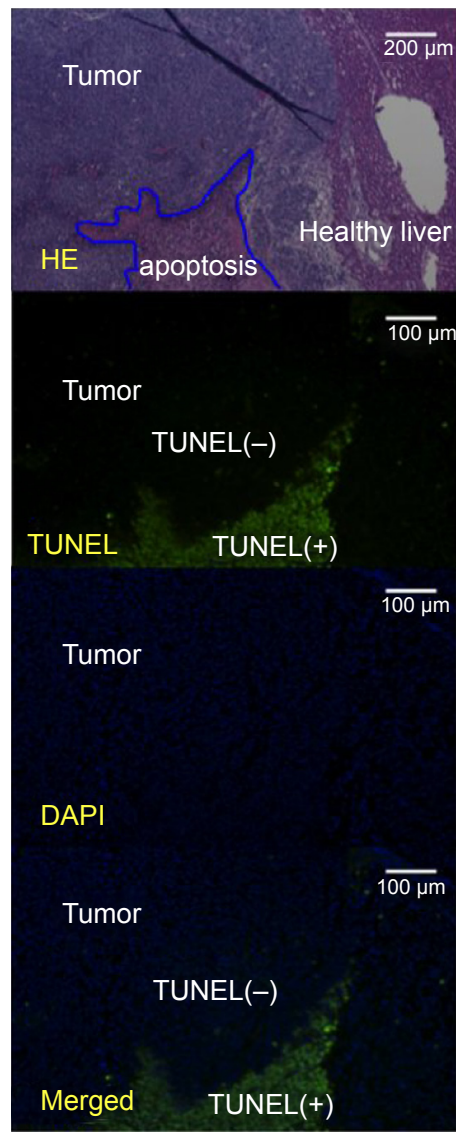

Figure 4 Images showing the tumors, histopathology, and tumor apoptosis.

Notes: (A) Macroscopic image of a tumor. (B, C) Microscopic characterization of an NI-SI hepatic tumor. Malignant cells with hyperchromatic nuclei, a high nuclear/ cytoplasmic ratio, and poor differentiation, along with hypervascularization and scattered necrotic regions. (D, E) Microscopic analysis of H\&E-stained tumor sections reveals two different regions: one containing typical malignant cells and the other containing disrupted cells characterized by missing nuclei, indistinct cell walls, or nuclei without surrounding cell structures. Upon collation with the observations from the fluorescent TUNEL assay, these disrupted cell regions were found to correspond with the regions with high levels of positive fluorescein-12-dUTP incorporation (TUNEL-positive regions).

Abbreviations: DAPI, 4',6-diamidino-2-phenylindole; H\&E, hematoxylin and eosin; TUNEL, terminal deoxynucleotidyl transferase dUTP nick-end labeling.

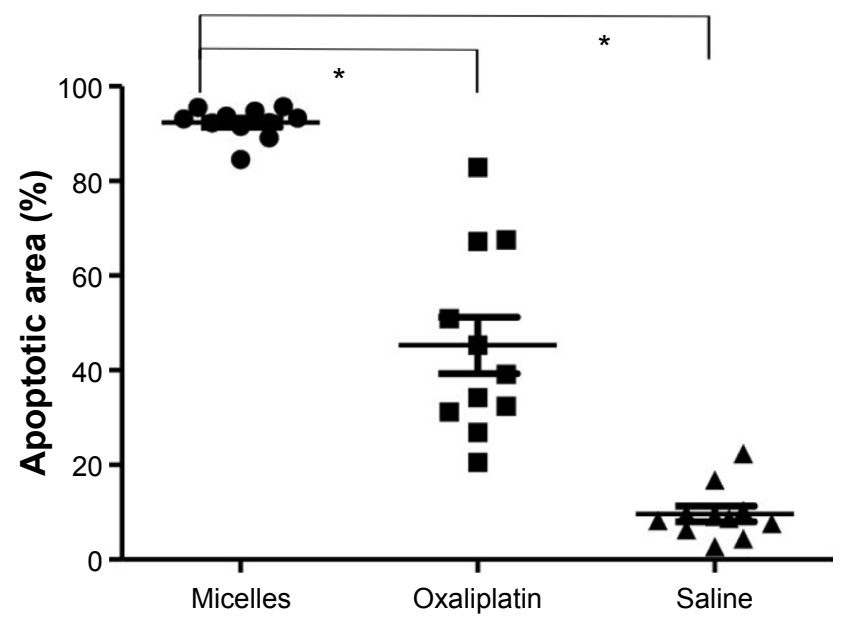

Figure 5 Apoptotic area percentages among the tumors at 3 days postinjection were $92.4 \% \pm 3.4 \%$ with the micelles $(n=10), 45.3 \% \pm 20.8 \%$ with oxaliplatin $(n=10)$, and $9.65 \% \pm 5.9 \%$ with saline $(n=10)$.

Notes: Gd DTPA/DACHPt-loaded micelles induced significant tumor cell death much more efficiently than free oxaliplatin and saline $(* P<0.00 \mathrm{I})$.

Abbreviations: DACHPt, (I,2-diaminocyclohexane)platinum(II); Gd-DTPA, gadoliniumdiethylenetriaminpentaacetic acid. were abdominally disseminated by 3 weeks postinjection. In contrast, there was no tumor suppression after oxaliplatin or saline treatment. With oxaliplatin, the mean tumor volume increased to $222 \mathrm{~mm}^{3}$ (129.0\% of the preinjection volume) at 3 days postinjection and to $997 \mathrm{~mm}^{3}(579.6 \%$ of the preinjection volume) at 10 days postinjection. Mean tumor volumes on day 14 were significantly larger in the oxaliplatin- and saline-injected rats than in the micelleinjected rats $(P<0.05)$. Moreover, the median postinjection survival of the micelle-treated rats (28 days) was significantly longer than those of the oxaliplatin-treated (20 days) and saline-injected rats (18 days) $(P<0.05)$ (Figure 7).

\section{Evaluation of systemic toxicity}

Following treatment with Gd-DTPA/DACHPt-loaded micelles, oxaliplatin, or saline, all rats survived to the end stage of cancer disease. No abnormal presentation was encountered during these treatments, with the exception of 


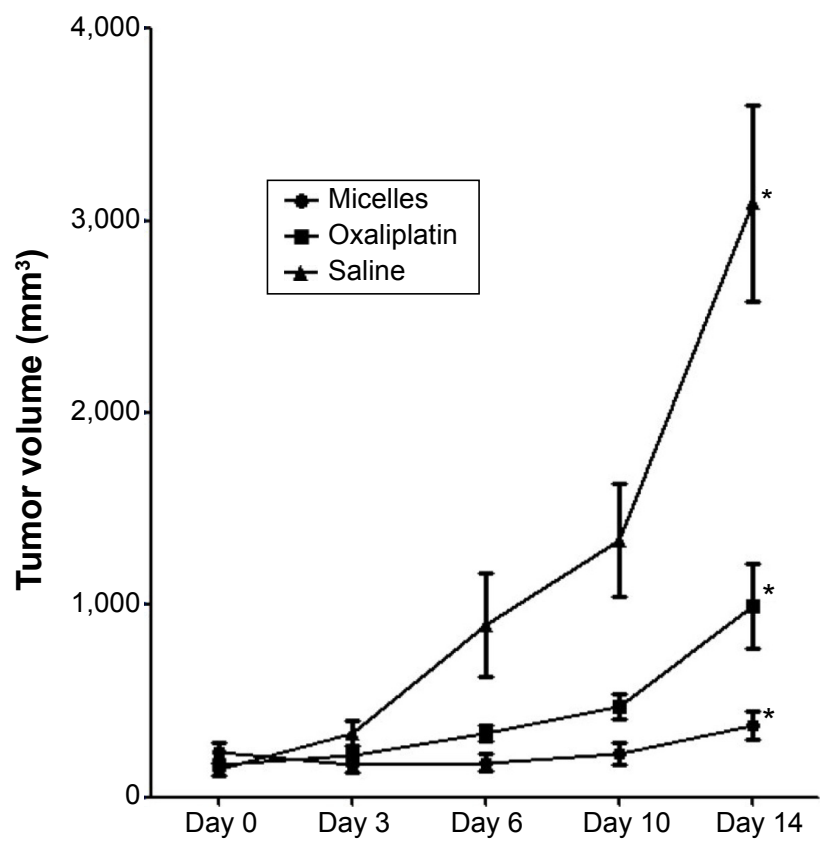

Figure 6 Tumor volume after the injection of micelles, oxaliplatin, or saline. Notes: At 3 days postinjection, the mean tumor volume of the micelle group decreased to $171 \mathrm{~mm}^{3}$ (74.0\% of the preinjection volume), and this tumor suppression continued until 10 days postinjection. The mean tumor volumes at day 14 were significantly larger in the oxaliplatin or saline injected rats than in the micelles injected rats $(* P<0.05)$. Values are shown as mean $\pm S D$.

Abbreviation: SD, standard deviation.

weight loss, which is summarized in Figure 8. The mean body weight of oxaliplatin-treated rats decreased by $14 \%$ and $22 \%$ by day 3 and day 7 , respectively, versus $10 \%$ and $13 \%$ in the micelle-treated rats. Systemic toxicity was evaluated by blood testing. Alanine aminotransferase levels increased after injection with the micelles and oxaliplatin, more so with oxaliplatin (Figure 9A). The alanine aminotransferase values from liver tissues for oxaliplatin injection may be even higher than those for micelle injection as micelles induced much higher rates of tumor cell death compared with oxaliplatin. Moreover, the Gd-DTPA/DACHPt-loaded micelle-treated rats exhibited a decrease in blood albumin levels at days 1 and 7 and an increase at day 14 (Figure 9B). In contrast, the oxaliplatin-treated rats exhibited severe decreases in blood albumin levels until day 14 . Thus, micelle injection caused a reversible hepatic dysfunction, while oxaliplatin caused a more severe and progressive disorder. Total bilirubin levels and renal function were not affected by either treatment (Figure 9C and D).

\section{Discussion}

Gd-DTPA/DACHPt-loaded micelles, when injected into the hepatic arteries of a rat model of HCC, provided strong and specific tumor contrast enhancement and induced high rates

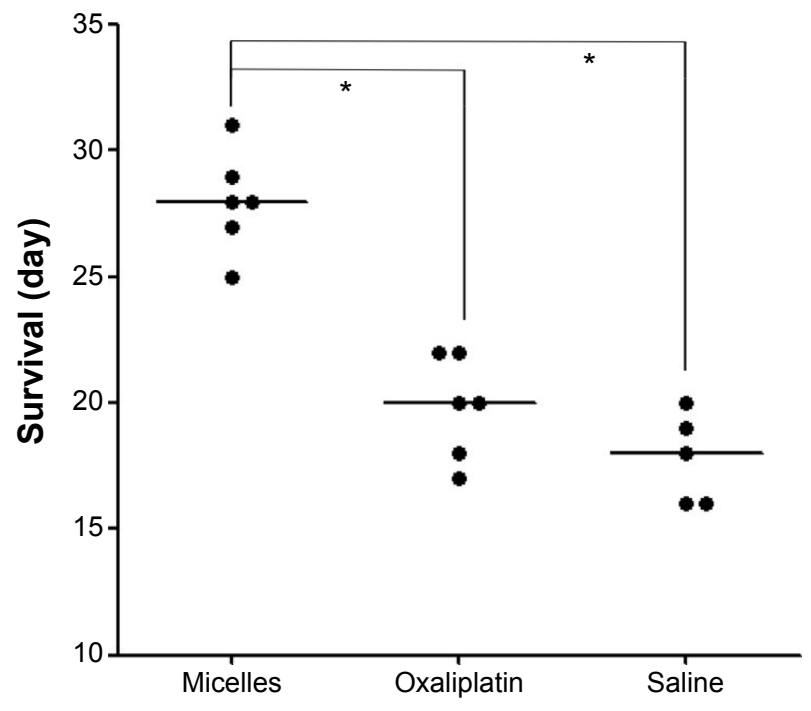

Figure 7 Survival outcomes after treatment.

Notes: The median survival duration after micelle injection (28 days) was significantly longer than those after oxaliplatin injection $(20$ days; $P *<0.05)$ and saline injection ( 8 days; $\left.P^{*}<0.05\right)$. Median values are shown with horizontal bars.

of tumor cell death, significantly reducing the tumor size and growth rate. Furthermore, the administration of micelles did not cause severe adverse reactions and improved survival outcomes in comparison with oxaliplatin or saline.

MRI is a useful method for the detection and characterization of regenerating and dysplastic nodules as well as HCC. ${ }^{34}$ Diagnostic sensitivity has been improved with

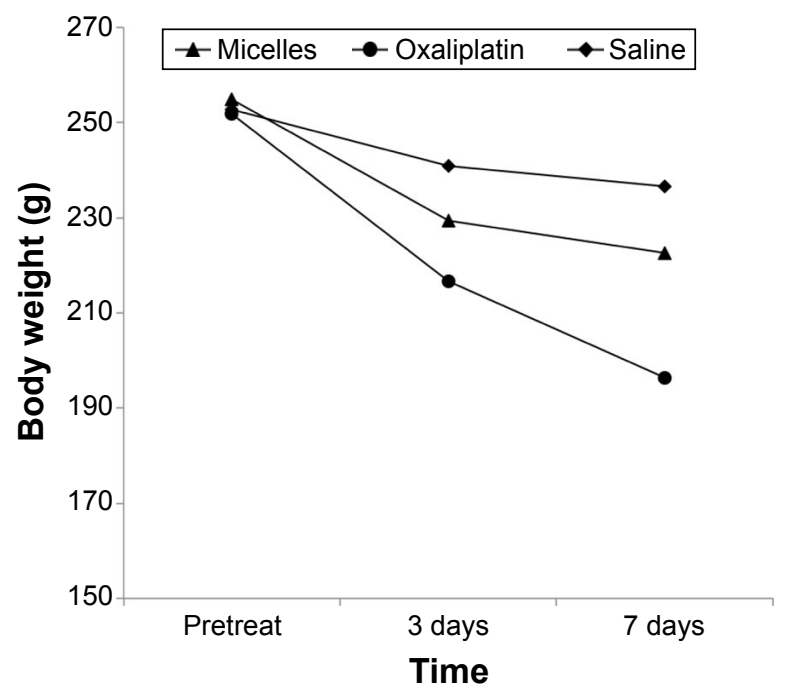

Figure 8 Body weight alternation following the injection of Gd-DTPA/DACHPtloaded micelles, oxaliplatin, and saline.

Notes: The injection of oxaliplatin caused the most severe weight loss among groups.

Abbreviations: DACHPt, (I,2-diaminocyclohexane)platinum(II); Gd-DTPA, gadoliniumdiethylenetriaminpentaacetic acid. 

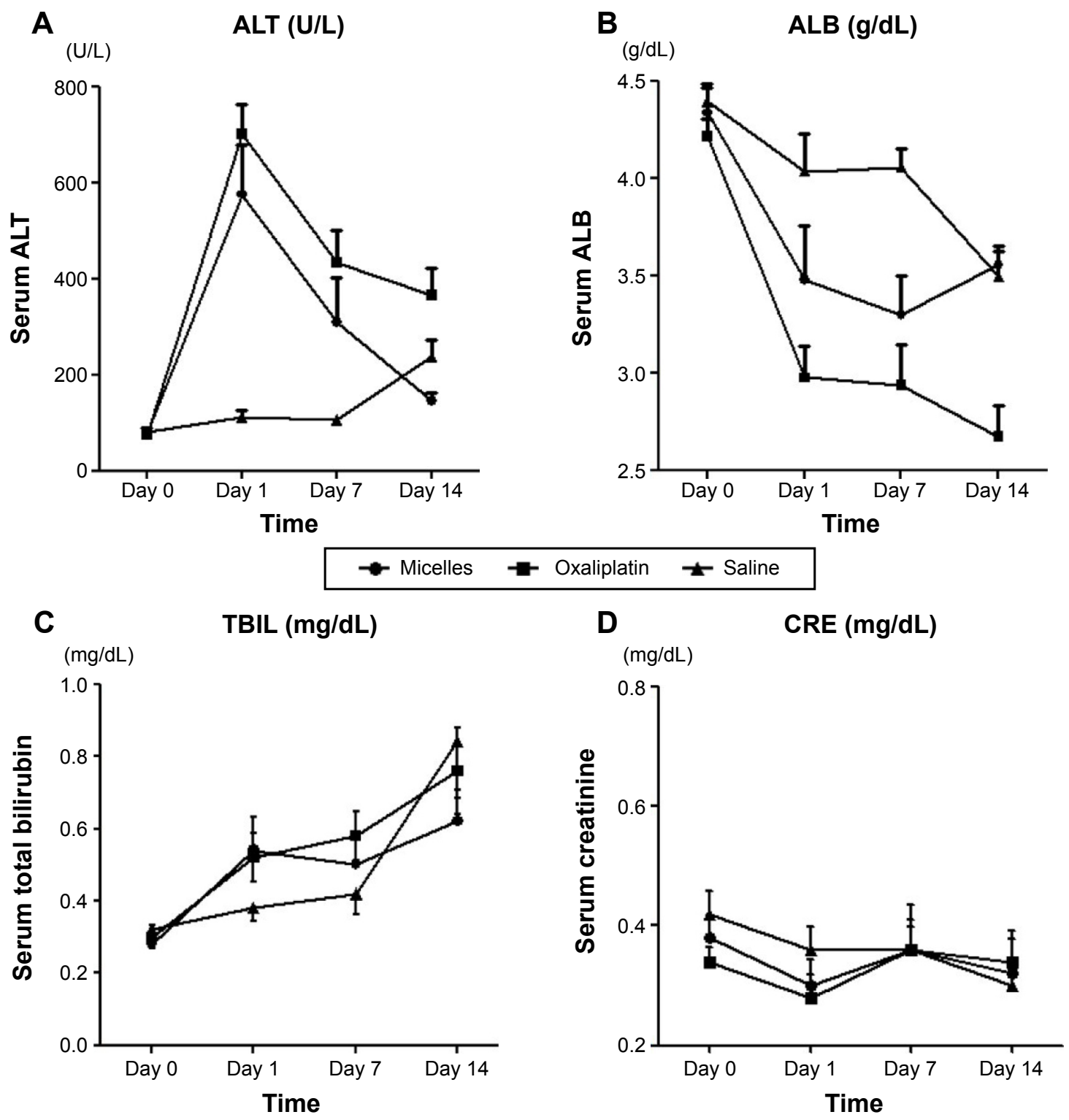

Figure 9 Blood test results.

Notes: (A) Alanine aminotransferase levels were increased after injection with both the micelles and oxaliplatin; however, more severe increases in alanine aminotransferase levels were observed after injection with oxaliplatin. (B) In the micelle-treated rats, blood albumin levels were decreased on days I and 7 and increased on day I4. In contrast, in the oxaliplatin-treated rats, blood albumin levels were markedly decreased until day 14. (C) Total bilirubin levels were not affected by either treatment. (D) Creatinine levels did not increase in all three groups.

Abbreviations: ALB, albumin; ALT, alanine aminotransferase; CRE, creatinine; TBIL, total bilirubin.

the use of contrast media, which enhance the contrast between lesions and the healthy liver and help to identify lesion characteristics. ${ }^{35}$ The dynamic enhanced acquisition technique after the administration of extracellular contrast agents is the most commonly used modality for diagnosing HCC. In this method, typical HCCs are characterized by early enhancement of signal intensity in the arterial phase followed by washout in the portal venous or delayed phases. The liver parenchyma is progressively enhanced, with maximum uptake occurring in the portal venous and equilibrium phases. ${ }^{36}$ However, dynamic phase imaging does not provide satisfactory contrast for the accurate diagnosis of hepatic lesions, especially small tumors. This is because the enhancement pattern depends on the structural properties of cells and tissues, such as the vascularity and permeability of the vascular endothelium. ${ }^{35,36}$ In this study, we incorporated an extracellular MRI contrast agent, Gd-DTPA, into the core of micelles to achieve tumor-selective targeting and retention based on the EPR effect. Gd-DTPA/DACHPt-loaded micelles did not release their contents in distilled water. However, under physiological condition $(10 \mathrm{mmol} / \mathrm{L}$ PBS at $37^{\circ} \mathrm{C}$ ), DACHPt and Gd-DTPA were released in a sustained 
manner; the release rate of DACHPt and Gd-DTPA was approximately $50 \%$ and $30 \%$ at 60 hours, respectively. ${ }^{17}$ Our results showed that the injection of Gd-DTPA/DACHPtloaded micelles modified the enhancement pattern in the liver. Tumor signal intensity was enhanced from hypointense to markedly hyperintense. In contrast, there was minimal change in the signal intensity in the healthy liver parenchyma, indicating that micelles did not permeate through normal vessels in healthy tissue. Furthermore, the high rate of tumor apoptosis and tumor growth suppression after treatment with Gd-DTPA/DACHPt-loaded micelles versus free drug supports the theory of high penetration, enhanced retention, and persistent and broad distribution within HCC tissues.

Cabral et al recently reported that size of sub-100 nm micelles affected the extravasation and penetration in poorly permeable tumors. ${ }^{37}$ The diameter of the Gd-DTPA/ DACHPt-loaded micelles was approximately $30 \mathrm{~nm}$, a size previously demonstrated to facilitate extravasation and tumor penetration in both hypervascular and poorly permeable hypovascular tumors; ${ }^{17,37,38}$ thus, we assumed that our micelles would be able to deeply penetrate HCC tissues. Moreover, Gd-DTPA/DACHPt-loaded micelles induced exceptionally bright contrast not only because of the accumulation of micelles in the tumor via the EPR effect, but also because of the augmentation of Gd-DTPA relaxivity in the cores of the micelles. ${ }^{17}$ These features may prove advantageous by improving the sensitivity of clinical HCC diagnoses, particularly in cases of multiple small tumors or satellite tumors in a cirrhotic liver, which may be overlooked by other imaging diagnostic modalities. Moreover, the concomitant incorporation of contrast molecules and anticancer agents in the micelles permits real-time evaluation of drug distribution in the tumors by MRI, thus enabling practitioners to adjust the doses to ensure proper efficacy with minimal adverse reactions and to anticipate treatment response.

Advanced HCC is one of the most intractable cancers, and resistance to conventional chemotherapy and radiotherapy and co-morbidities such as underlying chronic liver disease present major challenges in the development of effective therapies. Traditional therapeutics that feature systematic single or combination chemotherapies are extremely limited. Hepatic arterial intervention, another favorable method, includes non-arterial occlusion such as intra-arterial chemotherapy, lipiodolization, ie, lipiodol administration with or without antitumor drugs, or chemotherapy accompanied by arterial obstruction (chemoembolization). ${ }^{27,39-42}$ Nevertheless, only transcatheter arterial chemoembolization (TACE), in which the tumor artery should be selectively approached to limit access to the surrounding healthy liver, ${ }^{41,43}$ has been shown to improve survival in well-selected candidates. In TACE, anticancer agents should be solubilized or easily suspended in lipiodol and then gradually released in tumor. However, few antitumor agents have such properties. ${ }^{42}$ Our micelles provide a high drug-loading capacity even with hydrophobic substances and enable controllable release by engineering and modifying the micelles-forming block copolymers as well as multiple drug incorporation. These features are substantial advantages for developing effective drug carriers for HCC treatment. In addition, the tumor-targeting ability of the micelles may enhance the accumulation of their cargos in cancer tissue and limit their distribution to the healthy liver. In this study, Gd-DTPA/DACHPt-loaded micelles were non-selectively injected into the proper hepatic artery and produced an appreciable effect on diagnosis and treatment. This may prove to be a more feasible intervention than the selective arterial approach. Moreover, the use of micelles that provide selective MRI-contrast tumor enhancement facilitates disease management, as it allows patients and practitioners to avoid unnecessary exposure to ionizing radiation.

These findings highlight the capacity of the Gd-DTPA/ DACHPt-loaded polymeric micelles to safely provide remarkable MRI contrast enhancement and efficacy against HCC after administration through the hepatic artery. These results indicate that Gd-DTPA/DACHPt-loaded micelles are an excellent candidate for clinical translation for the diagnosis and treatment of HCC.

\section{Disclosure}

The authors report no conflicts of interest in this work.

\section{References}

1. Jemal A, Bray F, Center MM, et al. Global cancer statistics. CA Cancer J Clin. 2011;61(2):69-90.

2. Altekruse SF, McGlynn KA, Reichman ME. Hepatocellular carcinoma incidence, mortality, and survival trends in the United States from 1975 to 2005. J Clin Oncol. 2009;27(9):1485-1491.

3. Alves RC, Alves D, Guz B, et al. Advanced hepatocellular carcinoma. Review of targeted molecular drugs. Ann Hepatol. 2011;10(1):21-27.

4. Lencioni R. Chemoembolization for hepatocellular carcinoma. Semin Oncol. 2012;39(4):503-509.

5. Bruix J, Llovet JM. Prognostic prediction and treatment strategy in hepatocellular carcinoma. Hepatology. 2002;35(3):519-524.

6. Porta C, Paglino C. Medical treatment of unresectable hepatocellular carcinoma: going beyond sorafenib. World J Hepatol. 2010;2(3):103-113.

7. Matsumura Y, Maeda H. A new concept for macromolecular therapeutics in cancer therapy: mechanism of tumoritropic accumulation of proteins and the antitumor agent Smancs. Cancer Res. 1986;46:6387-6392.

8. Maeda $H$. The enhanced permeability and retention (EPR) effect in tumor vasculature: the key role of tumor-selective macromolecular drug targeting. Adv Enzym Regul. 2001;41(1):189-207. 
9. Cabral H, Nishiyama N, Kataoka K. Supramolecular nanodevices: from design validation to theranostic nanomedicine. Acc Chem Res. 2011; 44(10):999-1008.

10. Nishiyama N, Kataoka K. Current state, achievements, and future prospects of polymeric micelles as nanocarriers for drug and gene delivery. Pharmacol Ther. 2006;112(3):630-648.

11. Kataoka K, Harada A, Nagasaki Y. Block copolymer micelles for drug delivery: design, characterization and biological significance. Adv Drug Deliv Rev. 2001;47(1):113-131.

12. Torchilin VP. Recent advances with liposomes as pharmaceutical carriers. Nat Rev Drug Discov. 2005;4(2):145-160.

13. Duncan R. Polymer conjugates as anticancer nanomedicines. Nat Rev Cancer. 2006;6(9):688-701.

14. Davis ME, Chen Z, Shin DM. Nanoparticle therapeutics: an emerging treatment modality for cancer. Nat Rev Drug Discov. 2008;7(9): 771-782.

15. Kukowska-Latallo JF, Candido KA, Cao Z, et al. Nanoparticle targeting of anticancer drug improves therapeutic response in animal model of human epithelial cancer. Cancer Res. 2005;65(12):5317-5324.

16. Lee CC, MacKay JA, Frechet JMJ, Szoka FC. Designing dendrimers for biological applications. Nat Biotech. 2005;23(12):1517-1526.

17. Kaida S, Cabral H, Kumagai M, et al. Visible drug delivery by supramolecular nanocarriers directing to single-platformed diagnosis and therapy of pancreatic tumor model. Cancer Res. 2010;70(18):7031-7041.

18. Cabral H, Murakami M, Hojo H, et al. Targeted therapy of spontaneous murine pancreatic tumors by polymeric micelles prolongs survival and prevents peritoneal metastasis. Proc Natl Acad Sci US A. 2013;110(28): 11397-11402.

19. Sezgin Z, Yüksel N, Baykara T. Preparation and characterization of polymeric micelles for solubilization of poorly soluble anticancer drugs. Eur J Pharm Biopharm. 2006;64(3):261-268.

20. Trubetskoy VS. Polymeric micelles as carriers of diagnostic agents. Adv Drug Deliv Rev. 1999;37(1-3):81-88.

21. Rapoport N. Physical stimuli-responsive polymeric micelles for anticancer drug delivery. Prog Polymer Sci. 2007;32(8-9):962-990.

22. Matsumura Y, Hamaguchi T, Ura T, et al. Phase I clinical trial and pharmacokinetic evaluation of NK911, a micelle-encapsulated doxorubicin. Br J Cancer. 2004;91(10):1775-1781.

23. Matsumura Y. Preclinical and clinical studies of NK012, an SN-38-incorporating polymeric micelles, which is designed based on EPR effect. Adv Drug Deliv Rev. 2011;63(3):184-192.

24. Plummer R, Wilson RH, Calvert H, et al. A Phase I clinical study of cisplatin-incorporated polymeric micelles (NC-6004) in patients with solid tumours. Br J Cancer. 2011;104(4):593-598.

25. Kato K, Chin K, Yoshikawa T, et al. Phase II study of NK105, a paclitaxel-incorporating micellar nanoparticle, for previously treated advanced or recurrent gastric cancer. Investig New Drugs. 2012; 30(4):1621-1627.

26. Yokoyama M. Clinical applications of polymeric micelle carrier systems in chemotherapy and image diagnosis of solid tumors. J Exp Clin Med. 2011;3(4):151-158.

27. Yoshikawa M, Saisho H, Ebara M, et al. A randomized trial of intrahepatic arterial infusion of $4^{\prime}$-epidoxorubicin with Lipiodol versus $4^{\prime}$-epidoxorubicin alone in the treatment of hepatocellular carcinoma Cancer Chemother Pharmacol. 1994;33(1):S149-S152.
28. Xiao Y, Hong H, Javadi A, et al. Multifunctional unimolecular micelles for cancer-targeted drug delivery and positron emission tomography imaging. Biomaterials. 2012;33(11):3071-3082.

29. Nasongkla N, Bey E, Ren J, et al. Multifunctional polymeric micelles as cancer-targeted, MRI-ultrasensitive drug delivery systems. Nano Lett. 2006;6(11):2427-2430.

30. Murakami M, Cabral H, Matsumoto Y, et al. Improving drug potency and efficacy by nanocarrier-mediated subcellular targeting. Sci Transl Med. 2011;3(64):64ra2.

31. Cabral H, Nishiyama N, Okazaki S, Koyama H, Kataoka K. Preparation and biological properties of dichloro(1,2-diaminocyclohexane) platinum(II) (DACHPt)-loaded polymeric micelles. J Control Release. 2005;101(1-3):223-232.

32. Gouin S, Winnik FM. Quantitative assays of the amount of diethylenetriaminepentaacetic acid conjugated to water-soluble polymers using isothermal titration calorimetry and colorimetry. Bioconjug Chem. 2001;12(3):372-377.

33. Kishimoto S, Noguchi T, Yamamoto T, Fukushima S, Takeuchi Y. Antitumor effects of a novel lipophilic platinum complex (SM-11355) against a slowly-groing rat hepatic tumor after intra-hepatic arterial administration. Biol Pharm Bull. 2000;23(3):344-348.

34. Digumarthy SR, Sahani DV, Saini S. MRI in detection of hepatocellular carcinoma (HCC). Cancer Imaging. 2005;5(1):20-24.

35. Morana G, Salviato E, Guarise A. Contrast agents for hepatic MRI Cancer Imaging. 2007;7:24-27.

36. Bolog N, Andreisek G, Oancea I, Mangrau A. CT and MR imaging of hepatocellular carcinoma. J Gastrointestin Liver Dis. 2011;20(2): 181-189.

37. Cabral H, Matsumoto Y, Mizuno K, et al. Accumulation of sub-100 nm polymeric micelles in poorly permeable tumours depends on size. Nat Nano. 2011;6(12):815-823.

38. Rafi M, Cabral H, Kano MR, et al. Polymeric micelles incorporating (1,2-diaminocyclohexane)platinum (II) suppress the growth of orthotopic scirrhous gastric tumors and their lymph node metastasis. J Control Release. 2012;159(2):189-196.

39. Konno T, Maeda H, Iwai K, et al. Effect of arterial administration of high-molecular-weight anticancer agent SMANCS with lipid lymphographic agent on hepatoma: a preliminary report. Eur J Cancer Clin Oncol. 1983;19(8):1053-1065.

40. Kanematsu T, Inokuchi K, Sugimachi K, et al. Selective effects of lipiodolized antitumor agents. J Surg Oncol. 1984;25(3):218-226.

41. Bruix J, Sala M, Llovet JM. Chemoembolization for hepatocellular carcinoma. Gastroenterology. 2004;127(5):S179-S188.

42. Hanada M, Baba A, Tsutsumishita Y, et al. Intra-hepatic arterial administration with miriplatin suspended in an oily lymphographic agent inhibits the growth of tumors implanted in rat livers by inducing platinum-DNA adducts to form and massive apoptosis. Cancer Chemother Pharmacol. 2009;64(3):473-483.

43. Llovet JM, Bruix J. Systematic review of randomized trials for unresectable hepatocellular carcinoma: chemoembolization improves survival Hepatology. 2003;37(2):429-442.
International Journal of Nanomedicine

\section{Publish your work in this journal}

The International Journal of Nanomedicine is an international, peerreviewed journal focusing on the application of nanotechnology in diagnostics, therapeutics, and drug delivery systems throughout the biomedical field. This journal is indexed on PubMed Central, MedLine, CAS, SciSearch $®$, Current Contents $\AA /$ Clinical Medicine,

\section{Dovepress}

Journal Citation Reports/Science Edition, EMBase, Scopus and the Elsevier Bibliographic databases. The manuscript management system is completely online and includes a very quick and fair peer-review system, which is all easy to use. Visit http://www.dovepress.com/ testimonials.php to read real quotes from published authors. 